\title{
Reingeniería de enfermería en un hospital de segundo nivel: estrategias de gestión del cuidado
}

\author{
Wendy Melina Suasto-Gómez ${ }^{1 *}$, Irma Jiménez-Escobar ${ }^{2}$, Lasty Balseiro-Almario ${ }^{3}$, \\ Ana lilia Hernández-Hernández ${ }^{4}$, Adriana Chaparro-Hernández ${ }^{5}$ y Jessica Paulina Adán-Ruíz ${ }^{3}$ \\ ${ }^{1}$ Subdireccción de Enfermería, Hospital General Dr. Manuel Gea González; ${ }^{2}$ Dirección Médica, Hospital General Dr. Manuel Gea González; ${ }^{3}$ Escuela Nacional de \\ Enfermería y Obstetricia (ENEO), UNAM; ${ }^{3}$ Servicios Clínicos, Hospital General Dr. Manuel Gea González; ${ }^{5}$ Subdirección de Enseñanza e Investigación, Hospital General \\ Dr. Manuel Gea González. Ciudad de México, México
}

\begin{abstract}
Nursing re-engineering is a management strategy which fosters the review and re-design of processes to improve care management. This initiative begins in the Dr. Manuel Gea González General Hospital as a result of the new Nursing Sub-Direction for the period 2017-2022. The incorporation of a dedicated initiative on strategic planning has led to the gathering of important data to create groups of persons from diverse areas including services, teaching, management, and research, to help address the encountered problems and convey continuous improvements to the process of health care. (Hosp Med Clin Manag. 2019;12:91-7)

Corresponding author: Wendy Melina Suasto-Gómez, wendy_suasto@ hotmail.com
\end{abstract}

Key words: Nursing care. Management. Re-engineering.

\section{RESUMEN}

La reingeniería de enfermería es una metodología administrativa que permite la revisión y el rediseño de los procesos para lograr mejoras en la gestión del cuidado de las personas. La puesta en marcha de la reingeniería se inicia en el Hospital General Dr. Manuel Gea González a partir de la nueva gestión de la Subdirección de Enfermería para el periodo 2017-2022. La incorporación de la planeación estratégica ha permitido recopilar datos de importancia para formar grupos de personas de servicios, docencia, administración e investigación que permitan una mejora continua de los procesos a partir del análisis de los problemas encontrados.

Palabras clave: Cuidados de Enfermería. Gestión. Pacientes. Reingeniería.

\section{INTRODUCCIÓN}

El presente artículo tiene por objetivo analizar la reingeniería de enfermería en un hospital de segundo nivel como estrategia de gestión del cuidado a los pacientes. Para ello, se presentan a continuación el planteamiento de la tesis, la argumentación teórica, las conclusiones y la bibliografía.

\section{Correspondencia:}

*Wendy Melina Suasto-Gómez

E-mail: wendy_suasto @ hotmail.com
Date of reception: $23-10-2018$

Date of acceptance: 23-05-2019

DOI: 10.24875/HMCM.19000169 
Para Suasto ${ }^{1}$, la Subdirección de Enfermería se crea en el Hospital General Dr. Manuel Gea González en el año 1989, con las enfermeras Gloria Hernández García, Concepción Rosa Basurto y María de la Luz Maya. Para marzo de 1993 esta Subdirección queda a cargo de la enfermera Victoria Margarita Paz Sifuentes. Años después, en 2017, la Subdirección queda bajo la responsabilidad de la M.S.P. Wendy Melina Suasto Gómez, con la clara visión de otorgar servicios de enfermería de calidad con eficiencia y eficacia con personal altamente calificado, comprometido con el fomento a la salud y la prevención del daño, en un contexto profesional y ético, y con alto sentido humano, a la población usuaria. Por lo anterior, con el objeto de garantizar la mejora continua del personal ante las necesidades de salud de los usuarios, la Subdirección de Enfermería, apoyada en el Plan de trabajo 2017- 2022, inicia la reingeniería de enfermería con proyectos sustantivos en materia de servicios, docencia, administración e investigación, para beneficio del personal de enfermería y de las personas que este personal cuida.

\section{PLANTEAMIENTO DE LA TESIS: LA REINGENIERÍA DE ENFERMERÍA COMO ESTRATEGIA DE GESTIÓN DEL CUIDADO}

Para Lazcano, et al. ${ }^{2}$ La reingeniería en los servicios de enfermería representa una alternativa o forma de pensar para motivar y producir cambios radicales en las prácticas tradicionales, proporcionando cambios en las actitudes y valores en el personal de salud. Así, la reingeniería se puede entender como una metodología apropiada para reducir procesos, mejorar el servicio y mejorar la motivación y participación del personal. De igual forma, para Balderas ${ }^{3}$ la reingeniería de enfermería es la revisión y el rediseño radical de los procesos con la finalidad de lograr mejoras extraordinarias en cuanto a variables críticas de rendimiento, tales como calidad y servicios, entre otras.

\section{Puesta en marcha de la reingeniería}

Con base en la planeación estratégica, se inician los trabajos de reingeniería con el personal de enfermería para determinar mediante el análisis FODA (Fortalezas, Oportunidades, Debilidades y Amenazas) las acciones a seguir, entre las que destacan cuatro etapas:

Recopilación de datos. Aquí se señaló mediante un análisis cómo se están dando los procesos en los sistemas de organización de enfermería en servicios, docencia, administración e investigación. Para recopilar estos datos se reunió a jefes y supervisores de enfermería de todos los turnos de trabajo a fin de elaborar un diagnóstico situacional integral.

Motivación del personal. En esta etapa fue necesario presentar al personal de enfermería la problemática detectada y planear la reingeniería en cada uno de los proyectos de servicios, docencia, administración e investigación de enfermería, para establecer un diseño de mejora continua replanteando de manera drástica los cambios que se estiman pertinentes en la organización.

Formación de equipos de trabajo. En esta etapa se anotaron los nombres y apellidos de las personas integradas en la reingeniería aclarándoles la importante participación que tienen en lo que se desea alcanzar en los procesos de cambio. Desde luego, en cada equipo se nombró una líder que condujera los trabajos de los equipos.

Puesta en marcha. Los trabajos de reingeniería dieron inicio en junio del 2018, garantizando el rediseño de los procesos para la mejora continua de la calidad. Esto permite, según Hernández ${ }^{4}$, modificar los procesos básicos de trabajo en las organizaciones (Figs. 1 y 2).

\section{ARGUMENTACIÓN TEÓRICA DE LA TESIS: EL CUIDADO}

La enfermería del Hospital General Dr. Manuel Gea González tiene en esta nueva gestión (2017-2022) claramente definido su objeto de estudio, que es el cuidado, y su sujeto de estudio, que son las personas que comúnmente se llaman pacientes, usuarios o clientes. Estos cuidados son un acto de vida que, como plantea Colliere $^{5}$, también implica reciprocidad que se tiende a dar a cualquier persona que requiera temporal o definitivamente ayuda para cubrir sus necesidades vitales. Por ello, la enfermería renueva su filosofía en el desarrollo de un modelo de gestión del cuidado, y de una filosofía en misión, visión y valores de enfermería que permitan la identificación de la enfermería de la organización hospitalaria con lo que es ahora y lo que quiere lograr en un futuro: el bienestar de las personas, de las familias y de la comunidad en general. Lo que a continuación se presenta constituye la primera estrategia de gestión del cuidado para proporcionar bienestar a las personas. 


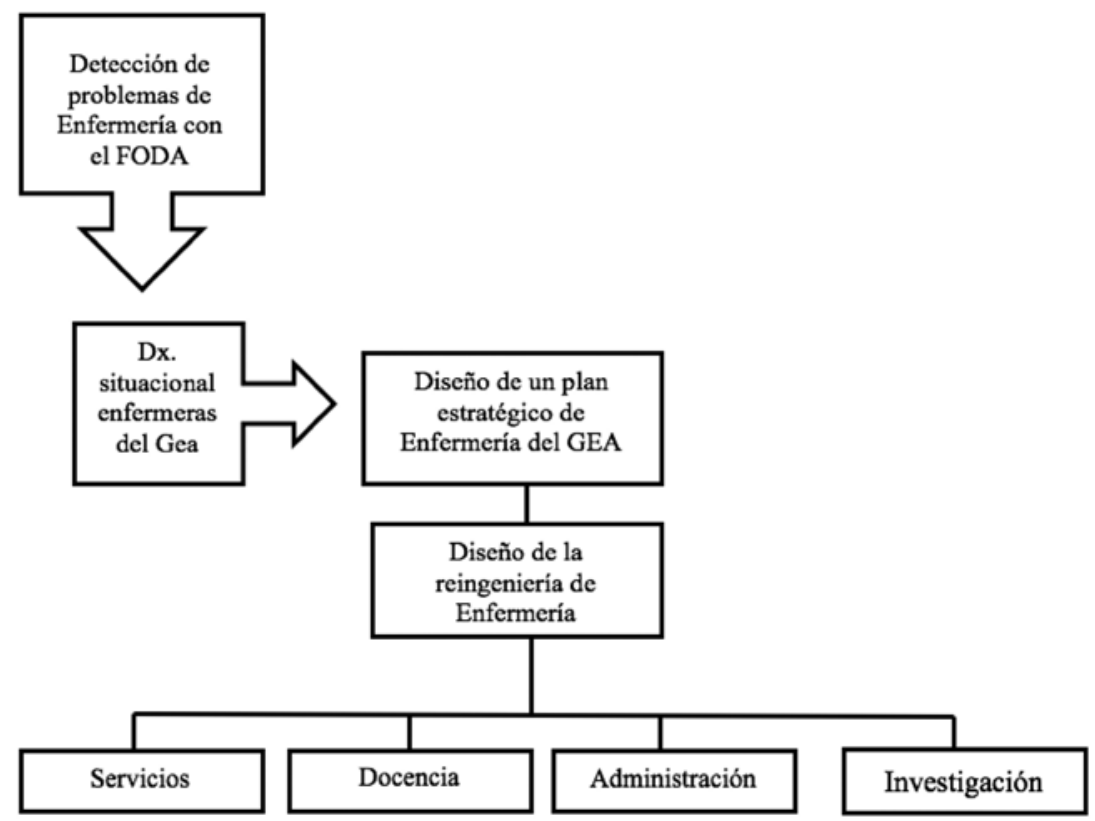

Figura 1. Plan estratégico de enfermería (adaptada de Suasto, $2018^{12}$ ). Diagnóstico FODA: fortalezas, oportunidades, debilidades y amenazas.

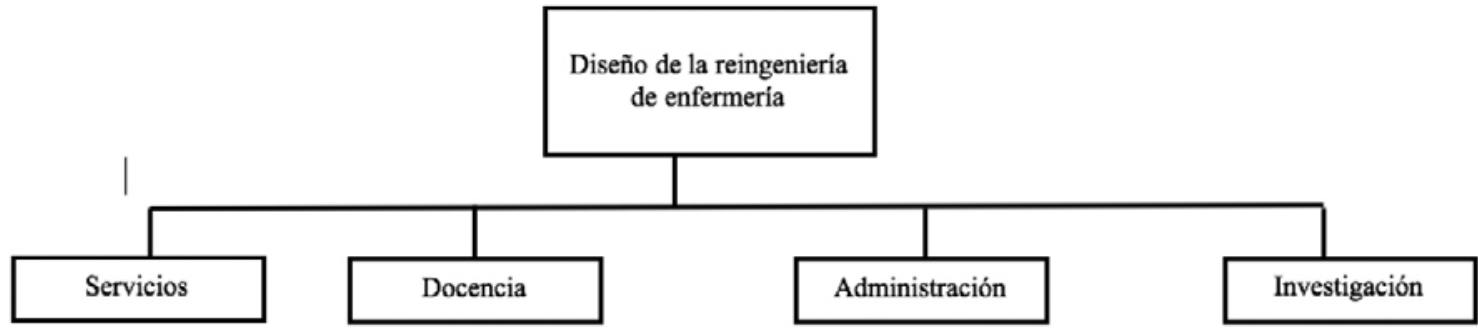

- Crear la filosofia de enfermería: misión, visión y valores

- Mejorar la organización en los Servicios

- Mejorar el ambiente de trabajo

- Contar con los insumos necesarios para el trabajo

- Establecer un programa de motivación personal

- Eficientar la enfermería

- Realizar sesiones clínicas de enfermería mensuales

- Crear un modelo de gestiór del cuidado de enfermería del Gea

- Redefinir funciones y actividades por nivel jerárquico
- Iniciar un diagnóstico situacional del NC

- Impartir seminarios, cursos y talleres de educación

- Mejorar y documentar la atención a Escuelas de Enfermería para campos clínicos

- Crear la profesionalización de nivel Licenciatura para Enfermeras Generales

- Iniciar la certificación profesional
- Revisar el Plan de trabajo de la Subdirección de Enfermería

- Documentar la planeación estratégica y la mejora continua

- Reorientar al personal al cumplimiento de metas

- Conformar un equipo de reingenieria de enfermeria

- Crear el proyecto de reingenieria

- Crear el proyecto: evaluación de los servicios de Enfermería

- Solicitar apoyo económico en la Fundación Río Arronte

- Crear el proyecto: Calidad en los servicios de enfermería

- Crear una revista: Enfermería del Gea

- Realizar foros académicos de investigación, docencia y administración de enfermería

- Generar artículos científicos

- Crear el proyecto: Evaluación de la supervisión de enfermería

- Crear un centro nacional de investigación de enfermería del Gea

- Impartir un curso de redacción de artículos científicos

Figura 2. Diseño de la reingeniería de enfermería (adaptada de Suasto, 2018 ${ }^{12}$ ). 


\section{Modelo de gestión del cuidado}

Un modelo es la representación gráfica de una realidad práctica, que se construye a partir de involucrar todas las gestiones y diligencias que la enfermería realiza a través de las interacciones, adaptaciones y adecuaciones de factores y recursos para brindar el cuidado. Si bien, según Kerouac, et al. ${ }^{6}$, existen modelos de gestión de las teóricas de la Enfermería como Dorothea Orem, Imogene King, Madeleine Leininger, Virginia Henderson y Calixta Roy, entre otras, que aseguran que administrar los cuidados requiere también de personal de enfermería con un conocimiento profundo de los diversos sistemas y subsistemas hospitalarios, así como del entorno, para producir el bienestar, este nuevo modelo de gestión de enfermería que se propone es de la siguiente manera: el modelo de gestión del cuidado de enfermería del Hospital General Dr. Manuel Gea González sienta sus bases en cuatro dimensiones: administración, salud, enfermería y el cuidado de la persona.

\section{Dimensión de administración}

Con relación a la dimensión de administración, el modelo plantea como base de sustentación el proceso administrativo con planeación, organización, integración, dirección y control, creado por Henry Fayol, «el padre de la Administración». De igual forma, a partir de esta interacción de las etapas del proceso, las enfermeras y los enfermeros que cuidan a las personas realizan la gestión del cuidado con un proceso continuo de interacción para mover los factores humanos y los recursos físicos, materiales, financieros en el entorno y fuera de él, para favorecer el cuidado de las personas que viven experiencias de salud y enfermedad.

\section{Dimensión de salud}

La dimensión de salud que plantea el modelo de gestión del cuidado de enfermería del Hospital General Dr. Manuel Gea González también tiene el sustento de la teoría de Avedis Donabedian sobre insumos, procesos y productos referentes a la salud. Con este apoyo de insumos, procesos y productos, la enfermería gestora moviliza los factores humanos, físicos, materiales y financieros para brindar un cuidado de excelencia. Esto significa que la enfermería trabaja en estrecha relación con el equipo multidisciplinario: los servicios médicos, paramédicos, de diagnóstico y tratamiento y los servicios generales, estableciendo relaciones profesionales con base en la interacción, coordinación y adaptaciones necesarias para proporcionar los cuidados.

\section{Dimensión de enfermería}

La dimensión de enfermería aporta de manera específica la gestión y el cuidado. Con la gestión, la enfermería del Hospital General Dr. Manuel Gea González asegura la disponibilidad de todos los recursos en la atención y cuidados a las personas en cantidad y calidad suficientes para brindar un cuidado seguro, óptimo y eficaz. Así, el cuidado es un valor que la enfermería produce a las personas sanas o enfermas mediante un proceso de interacción entre la enfermería y las personas que se cuidan para apoyar y ayudar. Para ello, las y los enfermeros se basan en el metaparadigma de enfermería, que es: la persona, la salud, el entorno y el cuidado que brinda la enfermería utilizando siempre un enfoque centrado en las necesidades de salud de las personas. El otro instrumento básico de cuidado que la enfermería brinda es el proceso de atención de enfermería, que implica la valoración, el diagnóstico de enfermería, la planeación de actividades de atención y la evaluación de los cuidados.

El modelo de gestión del cuidado también sienta sus bases en tres teóricas de enfermería, Imogene King, Virginia Henderson y Dorothea Orem. En el caso de Imogene King ${ }^{7}$, la organización hospitalaria constituye la parte más importante del entorno de cada persona que se cuida. Por ello, la enfermería del Hospital Gea González tiene un conocimiento profundo de los diversos sistemas que componen el entorno y las necesidades de las personas. Esto significa que, en la interacción, la enfermería utiliza también la teoría de sistemas de Von Bertalanffy, en donde existen microsistemas de atención, subsistemas internos de interrelación de las enfermeras con otros equipos de salud, y microsistemas del entorno y de la sociedad. Con esta interacción, se hace posible amalgamar el proceso de atención de enfermería, el proceso administrativo y las grandes funciones de enfermería, que son servicios, docencia, administración e investigación, para poder interactuar con los diversos comités: Infecciones nosocomiales, Ética, Quejas, Calidad, Mortalidad, Farmacia y Terapéutica, Subcomité de Expediente Clínico, Trasplantes y los comités administrativos (Adquisiciones, Arrendamiento y Servicios), así como también los diversos servicios paramédicos y generales del hospital para garantizar en esta interacción el cuidado de enfermería en un entorno 


\section{MODELO DE GESTION DEL CUIDADO DE ENFERMERIA DEL HOSPTTAL GENERAL} DR. MANUE GEA GONZALET.

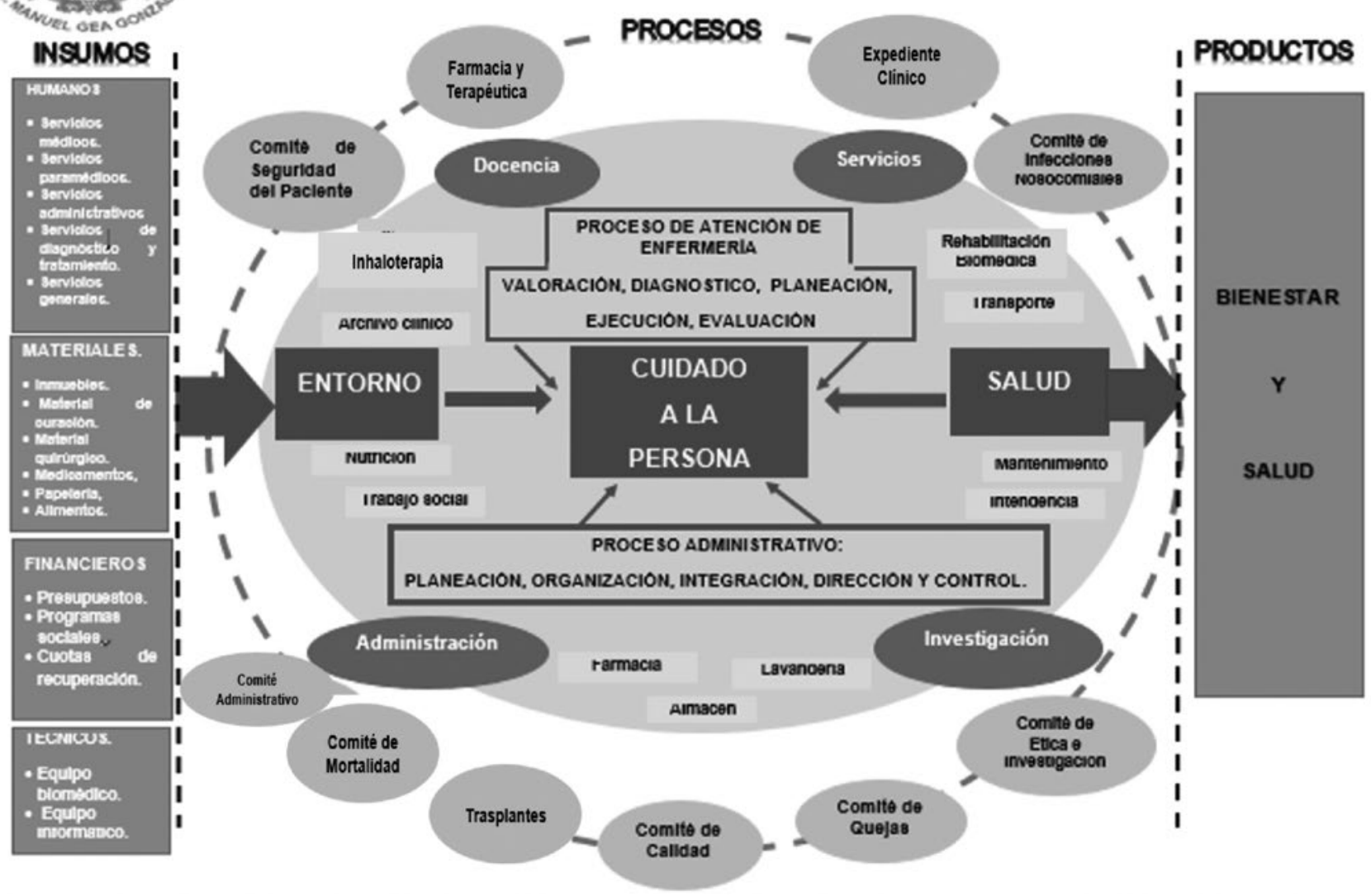

Figura 3. Modelo de gestión de enfermería del Hospital Gea González (adaptada de Suasto, 2018 ${ }^{12}$ ).

organizacional y social. Esta perspectiva social permite a la enfermería del Gea tomar las decisiones primordiales para brindar los cuidados centrados en el bienestar de las personas.

Otros conceptos utilizados son de la teórica de enfermería Virginia Henderson ${ }^{7}$, porque el modelo de gestión está centrado en las necesidades humanas de las personas que se cuidan. Esto significa que, para la enfermería, las personas son seres integrales con componentes biológicos, psicológicos, socioculturales y espirituales que interactúan entre sí, en donde el objeto de la enfermería es el cuidado y en donde los sujetos son la persona, la familia y la comunidad.

La base del sustento del cuidado también es de Dorothea Orem, porque la gestión debe asegurar la disponibilidad de los recursos para la atención de los usuarios y es allí donde la enfermera gestora establece la calidad y cantidad del personal y sus recursos para proveer una estructura de organización interactiva centrada en las necesidades de salud de las personas. Con esta visión clara de lo que es la práctica de cuidar, la enfermería del hospital garantiza los cuidados de calidad, seguros y óptimos que favorecen el bienestar de las personas. La idea principal de la enfermería, entonces, es considerar que cuando el paciente egrese del hospital, se cuide a sí mismo de tal forma que mantenga su salud y su bienestar (el modelo de gestión se muestra en la Fig. 3).

\section{La misión de enfermería}

Para Morales ${ }^{8}$, la misión es el motivo, el propósito, fin o razón de ser de la existencia de una empresa u organización para definir lo que se pretende cumplir en un entorno o sistema social en el que actúa, lo que pretende hacer y para quién lo va a hacer. Según Thompson $^{9}$, la misión describe razonablemente quiénes somos, qué hacemos y por qué estamos aquí. De hecho, 
para David ${ }^{10}$, la misión es una declaración duradera de objetivos que distinguen a una organización de otras similares. Es también la razón de ser de una empresa. Así, la misión de enfermería se deriva de la misión del hospital, promulgada en el Programa de Trabajo 2018 del Dr. Octavio Sierra Martínez ${ }^{11}$, que a la letra dice: «Brindar servicios de salud centrados en el paciente, desarrollando programas medicoquirúrgicos enfocados a la prevención, tratamiento y rehabilitación, con ética, equidad, calidad y seguridad, formando talento humano de excelencia e innovando con investigación aplicada y fortaleciendo redes interinstitucionales en un marco de eficiencia y efectividad».

Por lo anterior, la misión o razón de ser que se propone la enfermería del Hospital Dr. Manuel Gea González es la siguiente: «La razón de ser de la enfermería del Hospital General Dr. Manuel Gea González es brindar cuidados a las personas sanas, enfermas o que tengan carencias de salud de alguna índole. Este cuidado se brinda con la más alta calidad y calidez humana. Para realizar este cuidado la enfermería del Hospital General Dr. Manuel Gea González despliega sus conocimientos y su desempeño profesional en los servicios clínicos, de docencia, de administración y de investigación a fin de satisfacer las necesidades de bienestar y salud de las personas» ${ }^{12}$.

«En materia de servicios clínicos, se brindan cuidados de enfermería seguros y eficaces que garantizan el bienestar físico, mental, espiritual y social de individuos, familia y comunidades. Con relación a los servicios docentes, la enfermería se mantiene en continua capacitación para brindar cuidados generales y especializados de enfermería a las personas que lo requieran. Esta capacitación es dual, no solo para la enfermería sino también para las personas que se cuidan y sus familias».

«En el campo de la administración, la enfermería del Hospital General Dr. Manuel Gea González produce bienestar basado en el modelo de gestión de la Subdirección de Enfermería ${ }^{12}$, al interactuar y coordinar sus acciones con los diferentes servicios médicos, paramédicos, de diagnóstico, de tratamiento y servicios generales del Hospital. Esta interacción y coordinación que tiene la enfermería se hace necesaria y vital para proporcionar los cuidados de calidad que las personas sanas o enfermas esperan. De igual forma, en el campo de la investigación, la enfermería mantiene la actividad heurística de manera continua al incorporar con la metodología de la ciencia, proyectos e investigaciones y publicaciones de las diversas patologías y las intervenciones de enfermería en beneficio de las personas. Para ello, la enfermería produce intervenciones sistematizadas mediante la instrumentación del proceso de atención de enfermería para proporcionar calidad de la atención y calidez humana a fin de garantizar la satisfacción de la necesidad de la salud de las personas».

\section{La Visión de enfermería}

La visión para Hill y Jones ${ }^{13}$ es una declaración formal de lo que la empresa trata de lograr. Es decir, articula junto con la misión el intento estratégico de una compañía. Así, la visión es una declaración o manifestación escrita que indica hacia dónde se dirige una empresa o qué es aquello en que se pretende convertir a largo plazo. Para no confundir la misión con la visión, una forma sencilla de diferenciar estas dos declaraciones es recordando que la misión responde a la pregunta «¿cuál es nuestra razón de ser?», mientras que la visión responde a la pregunta "¿qué quieres llegar a ser?».

La visión de enfermería también se deriva de la visión propuesta por Sierra ${ }^{11}$ en su Programa de Trabajo 2018, que a la letra dice: "Ser una institución de salud, líder nacional e internacional en brindar servicios médicos de calidad, con educación de excelencia para el desarrollo de talentos e investigación innovadora, orientados a beneficios y compromiso social».

Por lo anterior, y derivado de esta visión del Hospital, la visión de enfermería que se propone es la siguiente:

«Vemos a la enfermería del Hospital General Dr. Manuel Gea González en el 2050 como los líderes nacionales en materia de cuidados integrales a las personas, mediante la utilización de tecnologías del cuidado vanguardistas que garanticen el cumplimiento de los objetivos institucionales y el bienestar de las personas sanas o enfermas» 12 .

\section{Los valores de enfermería}

Los valores, según Thompson, et al. ${ }^{9}$, son las creencias, características y normas conductuales que la administración determina deben guiar el cumplimiento de la misión y visión. los valores se relacionan con un trato 
justo, conducta, ética, trabajo en equipo, calidad suprema y responsabilidad social, entre otros.

Los valores de una organización son cualidades, principios o creencias que una empresa posee y que guían u orientan las decisiones, acciones y conductas de sus miembros. Los valores suelen enlistarse junto a la declaración de la misión y de la visión, para que los trabajadores los recuerden constantemente. el decálogo de valores de enfermería que se propone es la cuarta estrategia de la reingeniería en la gestión del cuidado y queda de la siguiente forma: «Trato digno a las personas, cuidados de excelencia, calidez humana, equidad de género, trabajo en equipo, seguridad en la atención, aplicación de tecnología en los cuidados de enfermería, compromiso social, bienestar y salud a las personas, desarrollo humano y profesional de enfermería».

\section{CONCLUSIONES}

La gestión del cuidado consiste en planear, organizar, dirigir y controlar los factores humanos y recursos físicos, materiales y financieros, para atender de la mejor manera posible los objetivos de una organización hospitalaria y brindar bienestar a las personas, las familias y las comunidades.

Para lograr lo anterior se requiere de un capital humano de enfermería que permita garantizar cuidados de calidad con la óptima utilización de los factores y recursos disponibles para cuidar, apoyar y atender a las personas y, con ello, cumplir cabalmente con los objetivos de la organización. Esto se conoce y se aplica en el Hospital General Dr. Manuel Gea González en donde, a pesar de contar con un entorno de salud marcado por continuos desafíos, el personal de enfermería multiplica sus cuidados mediante la reingeniería de procesos, en los turnos de trabajo, para beneficio de todas las personas sanas o enfermas que tienen alguna carencia de salud física, emocional, espiritual o social.

\section{AGRADECIMIENTOS}

Se deja constancia de nuestro agradecimiento al personal de enfermería de los Servicios, entre los que están jefes y supervisores, por su decidido entusiasmo en la reingeniería para la búsqueda de la mejora continua de las tecnologías del cuidado.

\section{CONFLICTO DE INTERESES}

Los autores declaran no tener conflicto de intereses en esta publicación.

\section{BIBLIOGRAFÍA}

1. Suasto WM. Plan de trabajo para la Subdirección de Enfermería 2017-2022. Hospital General Dr. Manuel Gea González. México: Secretaría de Salud; 2017.

2. Lazcano Ortiz M, Sánchez Moreno C, Jiménez Sánchez RC, Hernández Tapia E, Mejía Venado J, Torres Sandoval O. Reingeniería en los servicios de enfermería [Internet]. Universidad Autónoma del Estado de Hidalgo. Educación y salud; 2018 [Consultado el 30 de septiembre del 2018]. Disponible en: https://www.uaeh.edu.mx/scige/boletin/icsa/n3/m1.html

3. Balderas ML. Administración de los servicios de enfermería. En: Gómez D, editor. Gestión de vértice en salud: una visión estratégica de Enfermería, 2. ${ }^{a}$ ed. México: Universidad Autónoma del Estado de México; 2018.

4. Hernández C. Reingeniería: una herramienta para el trabajo administrativo. Ciencia administrativa N. ${ }^{0}$ 2. México: Instituto de Estudios Superiores de la Ciencia Administrativa, Universidad Veracruzana; 2012

5. Colliere MF. Promover la vida. 2. ${ }^{a}$ ed. México: Mc Graw Hill; 2009

6. Kerouac S, Pepin J, Ducharme F. El pensamiento enfermero. Madrid: Elsevier-Masson; 2007.

7. Raile Alligood M, Marriner Tomey A. Modelos y teorías en enfermería. Madrid: Elsevier; 2015.

8. Thompson A, et al. Administración estratégica: Teoría y casos. México: Mc Graw-Hill; 2015.

9. Morales, M. Como construir una filosofía empresarial. Disponible en: http:// www.pymepresarial.com.mx. México, 2014. p. 1

10. Sierra MO. Misión del Hospital General Dr. Manuel Gea González. Programa

11. Anual de Trabajo 2018. México: Hospital General Dr. Manuel Gea González, Dirección General; 2018.

12. David FR. La gerencia estratégica. Bogotá: Legis; 1990

13. Suasto WM. Modelo de gestión del cuidado de enfermería. Seminario Permanente de Reingeniería en Enfermería, Hospital General Dr. Manuel Gea González, Ciudad de México, agosto de 2018.

14. Hill Ch, Jones G. Administración estratégica. Un enfoque integral: Teoría y 15. casos 3. ${ }^{a}$ ed. México: Mc Graw-Hill; 1996. 